\title{
Kawasaki Disease
}

National Cancer Institute

\section{Source}

National Cancer Institute. Kawasaki Disease. NCI Thesaurus. Code C34825.

A vasculitis characterized by inflammation of the arteries, particularly the coronary arteries. The vasculitis may lead to aneurysm formation and possibly, heart attacks. It affects young children who usually present with persistent high fever, redness of the mucous membranes of the mouth, redness of the palms and soles, skin rashes, lymphadenitis, and joint pain and swelling. 